\title{
Light scattering spectrum of a suspension of interacting Brownian macromolecules ${ }^{\text {a) }}$
}

\author{
George D. J. Phillies \\ Department of Chemistry, The University of Michigan, Ann Arbor, Michigan 48109
}

Peter R. Wills

Max-Planck-Institut für Moleculare Genetik, 1000 Berlin 33, West Germany

(Received 12 September 1980; accepted 6 April 1981)

The joint effect of direct and hydrodynamic interactions on the dynamic structure $\mathbf{S}(\mathbf{k}, \mathbf{t})$ of a solution of rigid macromolecules is examined. The initial slope dS/dt and initial curvature $\mathrm{d}^{2} \mathrm{~S} / \mathrm{dt}^{2}$ of $\mathbf{S}(\mathbf{k}, \mathbf{t})$ are obtained. The reference frame correction of Kirkwood et al. [J. Chem. Phys. 33, 1505 (1960)] is shown to be wave-vector dependent. Contrary to some previous results, we argue that the initial slope of $S(k, t)$ is partly due to direct interparticle interactions rather than being due entirely to free-particle Brownian motion.

\section{INTRODUCTION}

The spectrum $S(\mathbf{k}, t)$ of light scattered by a solution of interacting Brownian macromolecules has been the subject of extensive theoretical investigation. Since the work of Altenberger and Deutch, ${ }^{1} S(\mathbf{k}, t)$ for such systems has been understood to reflect the interplay of direct and hydrodynamic interactions between the macromolecules. Early theoretical discussions of one of $\mathbf{u s}^{2,3}$ concluded that light scattering measures the mutual (pair) diffusion coefficient $D_{m}$ rather than the tracer (single-particle) diffusion coefficient $D_{T} ; D_{m}$ and $D_{T}$ are not equal in concentrated solutions. ${ }^{4}$ A number of authors have calculated the concentration dependence of $D_{m}$ and $D_{T}$, often with special reference to effects visible to light scattering spectroscopy. ${ }^{5-18}$ Most of these calculations are based on some form of the $N$ particle Smoluchowski equation in which the important physical effects are the isolated-particle Brownian motion, the hydrodynamic coupling between particle displacements, the direct (e.g., electrostatic) forces between the macromolecules, and the hydrodynamic drag on the moving macromolecules.

In contrast to some of the aforementioned papers, Pusey ${ }^{19}$ has argued that the initial decay of $S(\mathbf{k}, t)$ is due entirely to single-particle Brownian motion, direct interaction between diffusing macromolecules having no appreciable effect on $d S(\mathbf{k}, t) / d t$ at small $t$. Using different arguments, one of $\mathbf{u s}^{20}$ has presented a demonstation which appears to show that light scattering spectroscopy actually measures $D_{T}$ rather than $D_{m}$, while the other of us $^{21}$ has argued that direct intermacromolecular interactions contribute to the drag coefficient $f$ of a macromolecule, an effect which is not implicit in most other analyses of mutual diffusion in concentrated solution. ${ }^{22}$

Given the already voluminous literature on this problem, it is important to emphasize what significant new results are obtained here. In particular, (i) A previously uncalculated wave vector dependence of the reference frame corrections (as discussed by Kirkwood et al. ${ }^{23}$ )

\footnotetext{
a This work supported in part by the National Science Foundation under Grant CHE-7920389.
}

is found. (ii) The second cumulant $K_{2}=\lim _{t \rightarrow 0} d^{2} \ln S(\mathrm{k}, t) /$ $d t^{2}$ of the spectrum is obtained, showing that the forces responsible for $K_{2}$ cannot be correctly computed by neglecting the initial motion of the macromolecules. (iii) It is shown that direct interactions do contribute to $D_{m} k^{2}$, and that light scattering does measure a pair rather than a single-particle diffusion coefficient.

In Sec. II, a general power series expansion for $S(\mathbf{k}, t)$ is introduced. Terms corresponding to the direct interaction contribution to $f$ are identified and will be analyzed in a separate paper. In Sec. III, the initial slope of $S(\mathbf{k}, t)$ is found; in Sec. IV, a calculation of the initial curvature $K_{2}$ of $S(\mathbf{k}, t)$ is presented. Our results are discussed in Sec. V.

\section{TIME EXPANSION OF THE DYNAMIC STRUCTURE FACTOR}

The dynamic structure factor $S(\mathbf{k}, t)$ for a group of $N$ particles is given in terms of the scattering vector $k$ and the particle positions $\mathbf{r}_{i}(t)$ by

$$
S(\mathbf{k}, t)=\frac{1}{N}\left\langle\sum_{i, j=1}^{N} \exp \left\{i \mathbf{k} \cdot\left[\mathbf{r}_{i}(\tau+t)-\mathbf{r}_{j}(\tau)\right]\right\}\right\rangle_{\tau},
$$

where \langle\rangle$_{\tau}$ represents a time average. Applying the definition of the velocity

$$
\mathbf{r}_{i}(t)-\mathbf{r}_{i}(0)=\int_{0}^{t} \mathbf{v}_{i}(s) d s
$$

assuming the equivalence of time and ensemble averaging, and expanding the exponential as a power series in time gives

$$
\begin{aligned}
S(\mathrm{k}, t)= & \frac{1}{N}\left\langle\sum_{i, j=1}^{N} \exp \left[-i \mathrm{k} \cdot r_{j}(0)\right] \exp \left[i \mathrm{k} \cdot r_{i}(0)\right]\right. \\
& \left.\times \sum_{n=0}^{\infty} \frac{1}{n !}\left[i \mathrm{k} \cdot \int_{0}^{t} \mathrm{v}_{i}(s) d s\right]^{n}\right\rangle .
\end{aligned}
$$

We consider here systems whose volume is far larger than the volume over which particle positions or velocities are correlated.

Equation (2.3) gives $S(k, t)$ as an implicit function of time. $S(\mathbf{k}, t)$ may be obtained as an explicit function of time by using the power series expansion 


$$
S(\mathbf{k}, t)=\sum_{q=0}^{\infty} A_{q} t^{q} / q !
$$

with

$$
\begin{aligned}
& A_{q}=\frac{1}{N} \lim _{t=0} \frac{d^{q}}{d t^{Q}}\left\langle a_{-k 0} a_{k t}\right\rangle, \\
& a_{k t}=\sum_{i=1}^{N} e^{i \mathbf{r} \cdot \mathbf{r}_{i}(t)} .
\end{aligned}
$$

In Eq. (2.3), four effects modify $v_{i}(s)$ :

(a) Stress fluctuations in the solvent create a random velocity field in the solvent, which moves the suspended solute particles:

(b) Stress fluctuations at the surface of each solute particle cause the solute molecules to move with respect to the surrounding solvent, ${ }^{24}$ a motion distinct from the Brownian motion terms described in (a).

(c) Mechanical forces between pairs of solute molecules cause the solute molecules to move with velocities $F_{i l} \xi_{i}$, where $F_{i l}$ is the force on particle $i$ due to $l$ and $\xi_{i}$ is the mobility of $i$.

(d) Ignoring inertia, the total force $\sum_{l} F_{i l}$ on each solute molecule is exactly balanced by the hydrodynamic friction $f_{i}=-\sum_{l} F_{i l}$. The forces $f_{i}$ set up velocity fields $\mathbf{v}_{m}=\mathbf{T}_{m i} \cdot \mathbf{f}_{i}$ in the surrounding solution, where $\mathbf{T}_{m i}$ is a hydrodynamic interaction tensor.

Effects (a) and (b) are responsible for the Brownian velocity $\mathbf{v}_{B i}(t)$, while effects $(c)$ and $(d)$ create the interactive velocity

$$
\mathbf{v}_{I i}(t)=\sum_{i} \mathbf{F}_{i l} \cdot\left(\boldsymbol{\zeta}_{i}-\mathbf{T}_{i l}\right)+\sum_{(2, m) \neq i} \mathbf{T}_{i m} \cdot \mathbf{F}_{m l},
$$

which combine as

$$
\mathbf{v}_{i}(t)=\mathbf{v}_{I i}(t)+\mathbf{v}_{B i}(t),
$$

as suggested by Pusey. ${ }^{1 \theta}$ In Eq. (2.6), the first sum includes the motion of particle $i$ due to direct forces on it as well as the flow fields set up at particle $i$ by the force which particle $i$ exerts on other particles. The second sum includes the flow fields occurring at particle $i$ due to direct forces between the other particles in the solution. $F_{i l}$ is a function of the interparticle separation $\mathbf{r}_{i l}$, while $\xi_{i}, \mathbf{T}_{i l}$, and $\mathbf{v}_{B i}$ depend on the complete configuration $\left\{\mathbf{r}^{N}\right\}$ of all the solute molecules.

Substituting Éqs. (2.6) and (2.7) into Eq. (2.3), we define

$$
\begin{aligned}
J_{m n}= & \frac{1}{N}\left\langle\sum_{i, j=1}^{N} \exp \left[-i \mathrm{k} \cdot \mathrm{r}_{j}(0)\right] \exp \left[i \mathrm{k} \cdot \mathbf{r}_{i}(0)\right]\right. \\
& \left.\times \frac{1}{m ! n !}\left[i \mathrm{k} \cdot \int_{0}^{t} \mathrm{v}_{n i}(s) d s\right]^{m}\left[i \mathrm{k} \cdot \int_{0}^{t} \mathrm{v}_{B t}(s) d s\right]^{n}\right\rangle .
\end{aligned}
$$

We assume that the Brownian relaxation time $\tau_{B}$ is much less than other times of interest, in particular that $\tau_{B} \ll t$. We also take $t \ll\left(D_{0} k^{2}\right)^{-1}$, so that the positions of the particles at time $t$ are well approximated by their positions at $t=0$. The generalized Einstein relation ${ }^{20}$ gives

$$
\int_{0}^{t} d s_{1}\left\langle\mathbf{v}_{B i}\left(s_{1}\right) \mathbf{v}_{B i}(t)\right\rangle=\mathbf{D}_{i} .
$$

In considering the contribution of $J_{m n}$ to $A_{\theta}$, three pos sibilities arise:

(i) Some terms of $d^{4} J_{m n} / d t$ " contain factors of $i \mathrm{k} \cdot \int_{0}^{t} \mathrm{v}(s) d s$ without compensating factors of $\mathrm{v}(t)$. These terms vanish as $t-0$.

(ii) In other terms, the time derivatives cancel the integrals, leading to expressions like $\left[i \mathbf{k}^{\cdot} \mathrm{v}_{I i}(t)\right]^{m}$ $\times\left(-k \cdot D_{i} \cdot k\right)^{n / 2}$.

(iii) For $q>1$, there arise terms proportional to $d v_{i}(t) / d t$ which do not vanish in the limit $t \rightarrow 0$.

The terms of $A$ which remain are

$$
\frac{1}{N} \lim _{t \rightarrow 0} \frac{d}{d t}\left\langle a_{-k 0} a_{k t}\right\rangle=\lim _{t \rightarrow 0}\left\langle a_{-k 0} \sum_{i=1}^{N} e^{i \mathbf{r} \cdot \mathbf{r}_{i}(0)}\left[i \mathbf{k} \cdot \mathbf{v}_{I i}(t)+i \mathbf{k} \cdot \mathbf{v}_{B i}(t)-\int_{0}^{t} d s \mathbf{k} \cdot \mathbf{v}_{B i}(s) \mathbf{k} \cdot \mathbf{v}_{B i}(t)-2 \int_{0}^{t} d s \mathbf{k} \cdot \mathbf{v}_{B i}(s) \mathbf{k} \cdot \mathbf{v}_{I i}(t)\right]\right\rangle \text {. }
$$

Some care must be taken in interpreting the limit $t \rightarrow 0$. If one simply substitutes $t \equiv 0$, the right hand side of Eq. (2.10) vanishes; namely, in the ensemble average the velocity distribution function of each particle is independent of the positions of the other particles, and has the spherically symmetric Maxwell-Boltzmann form, so that an average of $\mathbf{k} \cdot\left[\mathrm{v}_{I i}(0)+\mathrm{v}_{B i}(0)\right]$ vanishes. The exact form of the term quadratic in $v_{B i}$ includes inertial effects, so for small $t$ it is of order $t^{1}$ and also vanishes if $t$ becomes identically equal to zero. However, we require that $t \gg \tau_{B} ; i \mathrm{k} \cdot \mathrm{v}_{B t}(t)$ is then equally likely to be positive or negative for each orientation of $\nabla_{B i}$, so in Eq. $(2.10)$ the term in $\left\langle i k \cdot v_{B i}(t)\right\rangle$ vanishes. The final term in Eq. (2.10), involving $\mathrm{k} \cdot \nabla_{B i}(s) \mathrm{k} \cdot \mathbf{v}_{I i}(t)$, corresponds to a contribution of direct interactions to the friction factor $f$. This term is dealt with elsewhere ${ }^{25}$; in the following we neglect all terms of this form. One notes that the lack of correlation between $\mathbf{v}_{B i}(t)$ and $\mathbf{v}_{B i}(0)$ does not preclude equal-time correlations between the Brownian velocities of different particles, corresponding to hydrodynamic interactions between the particles. The extent of these correlations is described by a two-particle relation analogous to Eq. (2.9). Equation (2.10) now becomes

$$
\begin{aligned}
& \frac{1}{N} \lim _{\substack{t \rightarrow 0 \\
\tau_{B} / t<1}} \frac{d}{d t}\left\langle a_{-k}(0) a_{k}(t)\right\rangle \\
& \quad=\frac{1}{N}\left\langle a_{-k 0} \sum_{i=1}^{N} e^{\left.i \mathbf{k}_{i 0}\left(i \mathbf{k} \cdot \mathbf{v}_{I i}-\mathbf{k} \cdot \mathbf{D}_{i} \cdot \mathbf{k}\right)\right\rangle .}\right.
\end{aligned}
$$


Several curiosities in the transition from Eq. (2.10) to Eq. (2.11) merit comment. First, at $t=0$ the canonical distribution function indicates that particle velocities have a spherically symmetric probability density. Since $\mathbf{v}_{I i}(0)$ [Eq. (2.6)] is nonvanishing, $\mathbf{v}_{B i}$ must also have a nonzero vector value at $t=0$, even though $\left\langle\mathbf{k} \cdot \nabla_{B i}\left(\tau_{B}\right)\right\rangle=0$. This discrepancy was resolved by Batchelor, ${ }^{11}$ who observed that for $t<\tau_{B}$ Brownian particles remember the direction to their location at $t=0$. If direct forces are present, the $N$-particle spatial distribution function is dependent on particle position, particles being more likely to be found in energetically favorable locations. At $t=0, \mathbf{v}_{B i}$ is correlated with the density of macroparticles, not because the fluid currents which drive $\nabla_{B i}$ have an average vector value, but because currents out of particle-rich regions move more particles than currents out of particle-poor regions. The particles' Brownian motions cause them to drift randomly away from their preferred positions, while the direct forces tend to drive them systematically back into those favored locations. The statement that $\mathbf{v}_{B i}$ and $v_{I i}$ cancel on the average at $t=0$ is equivalent to the better-known statement that the equilibrium $N$-particle spatial distribution functions are steady-state solutions to the $N$-particle Smoluchowski (diffusion) equation.

Equation (2.11) may also be written

$$
\begin{aligned}
A_{1}=\frac{1}{N}\left\langle a_{-k 0}\right. & \int_{a_{k 0}} d\{N\} \sum_{i=1}^{N} e^{i \mathbf{k} \cdot \mathbf{r}_{i}(0)}\left\{i \mathrm { k } \cdot \left[\sum_{l}\left(\zeta_{i}-\mathbf{T}_{i l}\right) \cdot \mathbf{F}_{i l}\right.\right. \\
& \left.\left.\left.+\sum_{(l, m) \neq i} \mathbf{T}_{i m} \cdot \mathbf{F}_{m l}\right]-\mathbf{k} \cdot \mathbf{D}_{i} \cdot \mathbf{k}\right\} e^{-\beta(W-A)}\right\rangle_{a_{k}},
\end{aligned}
$$

where

$$
\exp (-\beta A)=\int d\{N\} \exp (-\beta W)
$$

is a normalizing factor, and $W$ is the total potential energy of the solute. $\oint_{a_{0}} d\{N\}$ denotes integration over the hypersurface of configuration space for which

$$
\sum_{i=1}^{N} \exp \left[i \mathbf{k} \cdot \mathbf{r}_{i}(0)\right]=a_{k}(0),
$$

and \langle\rangle$_{a}$ indicates an average over possible values of $a_{k}(0),\left\langle\int d\{N\} a_{k}\right\rangle$ being taken over the isothermalisobaric ensemble.

\section{CONTRIBUTION OF DIRECT AND HYDRODYNAMIC FORCES TO $d S(k, t) / d t$}

The constrained integral of Eq. (2.12) is here evaluated by replacing it with an unconstrained integral whose integrand imposes the constraint. This is done by means of the approximation

$$
\begin{aligned}
\left\langle\left.\right|_{a_{k 0}}\right\rangle \equiv & \int_{a_{k 0}} d\{N\} e^{-\beta(W-A)} \Rightarrow \int d\{N\} e^{-\beta(W-A)} \\
& \times \exp \left(\sum_{j=1}^{N} \frac{a_{k 0} e^{-i k r_{j}}}{\left\langle\left|a_{k}\right|^{2}\right\rangle}\right),
\end{aligned}
$$

where $\left\langle I_{a_{k 0}}\right\rangle$ denotes the contrained average over all states with a fixed value of $a_{k 0}$ and

$$
\left\langle\left|a_{k}\right|^{2}\right\rangle=\sum_{i, j} \int d\{N\} e^{-\beta(W-A)} \exp \left[i \mathbf{k} \cdot\left(\mathbf{r}_{i}-\mathbf{r}_{j}\right)\right] .
$$

The substitution ( 3.1 ) is not altogether standard. In justification, we note that by definition

$$
\int_{a_{k 0}} d\{N\} e^{-\beta(W-A)}\left(\sum_{i=1}^{N} e^{i k \cdot r_{i}}\right)^{n}=a_{k 0}^{n} .
$$

The substitution (3.1) satisfies requirement (3.3) as long as the volume of the system is much greater than the volume within which particle positions are correlated. Furthermore, for $q \neq n k, n$ an integer, the approximation indicates

$$
\begin{aligned}
& \int_{a_{k 0}} d\{N\} e^{-\beta(W-A)}\left(\sum_{i=1}^{N} e^{i q \cdot \mathbf{r}_{i}}\right)=0, \\
& \int_{a_{k 0}} d\{N\} e^{-\beta(W-A)} \sum_{i, j=1}^{N} \exp \left[i \mathbf{q} \cdot\left(\mathbf{r}_{i}-\mathbf{r}_{j}\right)\right]=\left\langle\left|a_{a}\right|^{2}\right\rangle,
\end{aligned}
$$

as required by the independence of fluctuations in $a_{k}$ and $a_{q}$. The prescription (3.1) does not, however, correctly mirror effects due to mode-mode coupling terms, since it erroneously predicts

$$
\int_{a_{k 0}} d\{N\} e^{i q \cdot r_{i}-(k+q) \cdot r_{j}}=0
$$

Our previous results differed from Eq. (3.1) in two ways: (i) The product form of Ref. 21, i.e., $\Pi\left[1+a_{k 0} \exp \left(-i \mathbf{k} \cdot \mathbf{r}_{i}\right)\right]$ has been replaced by an exponential. (ii) The new normalizing factor $\left\langle\left|a_{k}\right|^{2}\right\rangle^{-1}$ appears in each term. The use of (i) gives Eq. (3.3) for $n>1$; the product form previously used by one of us ${ }^{21}$ does not supply the self-terms (e.g., $\left.\exp \left[-\mathbf{k} \cdot\left(\mathbf{r}_{1}+\mathbf{r}_{1}\right)\right]\right)$ needed for $n>1$. The normalizing factor $\left\langle\left|a_{k}\right|^{2}\right\rangle^{-1}$ has a physical interpretation: $a_{k}(0)$ may be increased either by changing the relative positions of distant particles or by distorting the most likely positions of the particles within clusters. The smaller $\left\langle\left|a_{k}\right|^{2}\right\rangle$ is, the harder it is to distort a cluster; as $\left\langle\left|a_{k}\right|^{2}\right\rangle$ becomes smaller, a given value of $a_{k}(0)$ corresponds more and more to the relocation of noninteracting particles:

$$
\begin{aligned}
A_{1}= & \frac{1}{N}\left\langle a_{-k 0} \sum_{i=1}^{N} \int d\{N\} e^{-\beta(W-A)} \exp \sum_{j=1}^{N} \frac{a_{k 0} e^{-i \mathbf{k} \cdot \mathbf{r}_{j 0}}}{\left\langle\left|a_{k}\right|^{2}\right\rangle} e^{i \mathbf{r} \cdot \boldsymbol{r}_{i 0}}\right. \\
& \left.\times\left\{i \mathrm{k} \cdot\left[\sum_{l}\left(\boldsymbol{\zeta}_{i}-\mathbf{T}_{i l}\right) \cdot \mathbf{F}_{i l}+\sum_{(i, m) \neq i} \mathbf{T}_{i m} \cdot \mathbf{F}_{m l}\right]-\mathbf{k} \cdot \mathbf{D}_{i} \cdot \mathbf{k}\right\}\right\rangle_{a_{k 0}} .
\end{aligned}
$$

Batchelor ${ }^{11}$ has shown that the general form for $D_{i}$ of a pair of spheres, with radius $a$, is

$$
\begin{aligned}
& \mathrm{D}_{i}=K_{B} T \zeta_{i}, \\
& \zeta_{i}=(6 \pi n a)^{-1}\left[1-\frac{15}{4}\left(\frac{a}{r}\right)^{4} \mathbf{r}+O\left(r^{-6}\right)\right],
\end{aligned}
$$


$\hat{r}$ being the unit vector along the line of centers. The trailing terms in $\boldsymbol{\zeta}_{i}$ represent modifications of the fluid flow around a sphere due to the other sphere; the mobilities parallel and perpendicular to $\hat{r}$ are not equal. Batchelor summarizes calculations for these terms for stick boundary conditions, including series expansions in inverse powers of the center-to-center separation $r$ of the spheres. Felderhof has extended these series to higher powers of $1 / r$ and generalized them to arbitrary stick-slip boundary conditions. ${ }^{12}$ To order $(a / r)^{4}$ the pair approximation for $\zeta_{i}$ in an infinite suspension of spheres is

$$
\zeta_{i}=\frac{1}{6 \pi \eta a}\left[1-\sum_{j \neq i} \frac{15}{4}\left(\frac{a}{r_{i j}}\right)^{4} \mathbf{r}_{i j} \mathbf{r}_{i j}\right] \text {. }
$$

At low concentrations at which only pairs of spheres interact, the final term of Eq. (2.12) is

$$
\left\langle\frac{1}{N}\left\langle\left. a_{-k 0} \sum_{i=1}^{N} e^{i \mathbf{r} \cdot r_{i}(0)}\left(-\mathbf{k} \cdot \mathbf{D}_{i i} \cdot \mathbf{k}\right)\right|_{a_{k 0}}\right\rangle\right\rangle_{a_{k 0}}=-D_{0} k^{2}\left\{S(k)-4 \pi c_{0} \int r^{2} d r g(r) \frac{\left[-2+(k r)^{2}\right] \sin (k r)+2 k r \cos (k r)}{(k r)^{3}} \frac{15}{4}\left(\frac{a}{r}\right)^{4}\right\},
$$

where $D_{0}=K_{B} T / 6 \pi \eta a, g(r)$ is the radial distribution function, and $c_{0}$ the number concentration.

The first part of Eq. (3.6) may also be reduced. Assuming that a given particle only interacts with one of its neighbors at a time, and that $W$ is a sum of pair potentials $W_{i j}$,

$$
\begin{aligned}
I_{1} \equiv & \frac{1}{N}\left\langle\left\langle\left. a_{-k 0} \sum_{i=1}^{N} e^{i \mathbf{k} \cdot \mathbf{r}_{i}(0)}\left[i \mathbf{k} \cdot \mathbf{v}_{I t}(0)\right]\right|_{a_{k 0}}\right\rangle\right\rangle \\
& =-\frac{1}{N} \sum_{i, j=1}^{N} \int d\{N\} \beta^{-1} \nabla \cdot\left[e^{i \mathbf{k} \cdot \boldsymbol{r}_{i j} j_{i} \mathbf{k}} \cdot\left(\boldsymbol{\zeta}-\mathbf{T}_{i j}\right)\right]\left(e^{-\beta(W-A)}-C\right),
\end{aligned}
$$

where $C$ is a constant of integration, to be chosen for convenience. For an incompressible solution, one is tempted to write ${ }^{1} \boldsymbol{\nabla}_{i} \cdot \boldsymbol{T}_{i j}=0$. However, unless the solute macroparticles displace no solvent, as assumed in Ref. 1, the volume current of solute $J_{i}$ into a closed volume must cancel the volume current of solvent $J_{s}$ flowing into the same volume. As shown in the Appendix, this leads to

$$
\sum_{j=1}^{N} \int_{v} d \mathbf{r}_{i}\left[\hat{\mathbf{k}} \cdot \mathbf{b}_{i j}^{\prime} \cdot \hat{\mathbf{k}} e^{i \mathbf{r} \cdot \mathbf{r}_{i j}}-\phi_{j} H(k)\right]=0
$$

where $b_{i j}^{\prime}$ is the true hydrodynamic interaction tensor for a closed volume, $\phi_{j}$ is the volume fraction of particle $j$ in the system, and $H(k)$ is the spatial Fourier transform of the particle shape.

The usual Oseen tensor form $\mathbf{T}^{\mathrm{Os}}$ for $\mathbf{b}_{i j}^{\prime}$ may be corrected for the nonzero extent of each particle, but neglecto solvent backflow by assuming that the solution has no boundaries. For intermediate particle separations $r_{i j}$, the numerical difference between $\mathbf{r}^{\mathrm{O}}$ and $\mathbf{b}_{i}^{\prime}$ is very small; however, integrals over $\mathbf{T}^{\mathrm{O}}$ or $\mathbf{b}_{i j}^{\prime}$ such as those in Eq. (3.11) are very different. ${ }^{17}$ Solvent backflow corresponds microscopically to the reference frame corrections of Kirkwood et al. ${ }^{24}$ For small $(r / a), b_{i}^{i}$ is approximated by its open volume form

$$
\mathbf{b}_{i j}=\left[\frac{3 a}{4 r_{i j}}\left(\mathbf{l}+\hat{\mathbf{r}}_{i j} \hat{\mathbf{r}}_{i j}\right)+\frac{1}{2}\left(\frac{a}{r_{i j}}\right)^{3}\left(1-3 \hat{\mathbf{r}}_{i j} \hat{\mathbf{r}}_{i j}\right)\right] \text {, }
$$

where $\mathbf{T}_{i j}=(6 \pi \eta)^{-1} \mathbf{b}_{i j}$. Integration by parts of Eq. (3.10), use of the approximate form (3.12), restriction to terms of lowest order in concentration, and addition of 0 [in the form of Eq. (3.11)] show

$$
\begin{aligned}
I_{1}= & D_{0} k^{2}\left[S_{D}(k)+\phi H(k)-4 \pi c_{0} \int r^{2} d r\left(\frac{4 k r \cos (k r)+\left[(k r)^{2}-4\right] \sin (k r)}{(k r)^{3}}\right.\right. \\
& \left.\left.\times \frac{15}{4}\left(\frac{a}{r}\right)^{4} g(r)+[g(r)-1]\left\{\frac{3 a}{2 r} \frac{\left[(k r)^{2}-1\right] \sin (k r)+k r \cos (k r)}{(k r)^{3}}-\left(\frac{a}{r}\right)^{3} \frac{\left[(k r)^{2}-3\right] \sin (k r)+3 k r \cos (k r)}{(k r)^{3}}\right\}\right)\right],
\end{aligned}
$$

where

$$
S_{D}(k)=\int d \mathbf{r} e^{\mathbf{n} \cdot \mathbf{r}}[g(r)-1]
$$

From Eqs. (3.9) and (3.13),

$$
\begin{aligned}
A_{1}= & -D_{0} k^{2}\left(1-\phi H(k)+4 \pi c_{0} \int_{0}^{\infty} d r r^{2}\left\{g(r) \frac{15}{2}\left(\frac{a}{r}\right)^{4}\left(\frac{X \cos X-\sin X}{X^{3}}\right)\right.\right. \\
& \left.\left.+[g(r)-1]\left[\left(\frac{3 a}{2 r}\right) \frac{\left(X^{2}-1\right) \sin X+X \cos X}{X^{3}}-\left(\frac{a}{r}\right)^{3} \frac{\left(X^{2}-3\right) \sin X+3 X \cos X}{X^{3}}\right]\right\}\right),
\end{aligned}
$$

where $X=k r$. In the limit $k \rightarrow 0$,

$$
A_{1}=-D_{0} k^{2}\left(1-\phi+4 \pi c_{0} \int_{0}^{\infty} d r r^{2}\left\{\frac{-5}{2}\left(\frac{a}{r}\right)^{4} g(r)+\frac{a}{r}[g(r)-1]\right\}\right),
$$

and if detailed hydrodynamic interactions between the particles are ignored, 


$$
A_{1}=-D_{0} k^{2}(1-\phi)
$$

\section{SECOND TIME CUMULANT OF $S(k, t)$}

We obtain the second time cumulant of the spectrum

$$
K_{2}=\lim _{t \rightarrow 0} \frac{\partial^{2}}{\partial t^{2}}\{\ln [S(\mathbf{k}, t)]\}
$$

from $d^{2} S(k, t) / d t^{2}$. The arguments which led to Eq. (2.11) give

$$
\begin{gathered}
\frac{1}{N} \lim _{\substack{t \rightarrow 0 \\
\tau_{B} / t<1}} \frac{d^{2}}{d t^{2}}\left\langle a_{-k}(0) a_{k}(t)\right\rangle=\lim _{t \rightarrow 0} \frac{1}{N}\left\langlea _ { - k 0 } \sum _ { i = 1 } ^ { N } e ^ { i \mathbf { k } \cdot \mathbf { r } _ { i 0 } } \left\{ i \mathbf{k} \cdot \frac{d \mathbf{v}_{I i}(t)}{d t}-\left[\mathbf{k} \cdot \mathbf{v}_{I i}(t)\right]^{2}-\int_{0}^{t} d s\left[\mathbf{k} \cdot \mathbf{v}_{i}(s) \mathbf{k} \cdot \frac{d \mathbf{v}(t)}{d t}\right]\right.\right. \\
\left.\left.\quad-i \int_{0}^{t} d s[\mathbf{k} \cdot \mathbf{v}(s)][\mathbf{k} \cdot \mathbf{v}(t)]^{2}+\frac{1}{2} \int_{0}^{t} \int_{0}^{t} d s_{1} d s_{2}\left[\mathbf{k} \cdot \mathbf{v}_{i}\left(s_{1}\right) \mathbf{k} \cdot \mathbf{v}_{i}\left(s_{2}\right) \mathbf{k} \cdot \mathbf{v}_{i}(t) \mathbf{k} \cdot \mathbf{v}_{i}(t)\right]\right\}\right\rangle
\end{gathered}
$$

where only nonvanishing terms, not dependent on the interactive contribution to the friction, have been retained.

The second, fourth, and fifth terms of Eq. (4.2) are products of terms encountered in Sec. III. Under the interacting pair approximation, these terms are

$$
I_{1}=\frac{1}{N}\left\langle\sum_{i, j=1}^{N} \int d\{N\} e^{i \mathbf{k} \cdot \mathbf{r}_{i j}}\left\{-\left[\mathbf{k} \cdot\left(\zeta_{i}-\mathbf{T}_{i j}\right) \cdot \mathbf{F}_{i j}{ }^{2}-2 i \mathbf{k} \cdot\left(\zeta_{i}-\mathbf{T}_{i j}\right) \cdot \mathbf{F}_{i j}\left(\mathbf{k} \cdot \mathbf{D}_{i} \cdot \mathbf{k}\right)+\left(\mathbf{k} \cdot \mathbf{D}_{i} \cdot \mathbf{k}\right)^{2}\right\} e^{-\mathbf{B}(W-A)}\right\rangle,\right.
$$

the limit $t \rightarrow 0$ being taken. Evaluation of these terms in the presence of detailed hydrodynamic interactions is tedious. In the absence of such forces, comparison with Sec. III shows

$$
\begin{aligned}
I_{1}= & \left(D_{0} k^{2}\right)^{2}\left\{S_{D}(k)-k^{-2} c_{0} \int d r g(r) e^{i \mathbf{k} \cdot \mathbf{r}} e^{-\boldsymbol{a w}}(\hat{\mathbf{k}} \cdot \hat{\mathbf{r}})^{2} \frac{\partial^{2} \beta W_{i j}}{\partial r^{2}}\right. \\
& \left.-2\left[S_{D}(k)+\phi H(k)\right]+S(k)\right\},
\end{aligned}
$$

which is comparable term by term with Eq. (4.3).

The first and third terms of Eq. (4.2) require slightly more careful treatment. Since $\tau_{B} \ll t$, averages over $d \mathrm{v}_{B} / d t$ vanish. The same is not true of the interactive velocity; with our usual approximations

$$
\frac{d \mathrm{v}_{I i}}{d t}=\left(\mathbf{v}_{i} \cdot \nabla_{i}+\mathbf{v}_{j} \cdot \nabla_{j}\right) \cdot\left(\frac{\mathbf{F}_{i j}}{f}\right) ;
$$

so the first and third terms of Eq. (4.2) become

$$
\begin{aligned}
I_{2}= & \left(D_{0} k^{2}\right)^{2} c_{0} \int d r g(\mathbf{r}) e^{i \mathbf{r} \cdot \mathbf{r}} \\
& \times\left\{\hat{\mathbf{k}} \cdot \hat{\mathbf{r}} \beta \mathbf{F}(\boldsymbol{r}) \cdot \nabla[\beta \mathbf{F}(\boldsymbol{r})] k^{-3}+(\hat{\mathbf{k}} \cdot \hat{\mathbf{r}})^{2} \frac{\partial^{2} \beta W}{\partial r^{2}} k^{-2}\right\} .
\end{aligned}
$$

Combining $I_{1}$ and $I_{2}$,

$$
\begin{aligned}
& \lim _{t \rightarrow 0} \frac{d^{2} S(\mathbf{k}, t)}{d t^{2}}=\left(D_{0} k^{2}\right)^{2}\{1-2 \phi H(k) \\
& \left.+c_{0} \int d \mathbf{r} g(r) e^{i \mathbf{r} \cdot \mathbf{r}} \hat{\mathbf{k}} \cdot \hat{\mathbf{r}} \beta \mathbf{F}(r) \cdot \nabla[\beta \mathbf{F}(r)] k^{-3}\right\} .
\end{aligned}
$$

From Sec. III, the first time cumulant of $S(k, t)$ is

$$
+K_{1} \equiv-\lim _{t \rightarrow 0} \frac{d \ln S(k, t)}{d t}=+\frac{D_{0} k^{2}[1-\phi H(k)]}{S(k)} .
$$

Neglecting terms in the square of the concentration, so that $[S(k)]^{2}-1=2 S_{D}(k)$ and $\phi S_{D}(k) \cong 0$, the second time cumulant is $A_{2} / S(k)-\left[K_{1}\right]^{2}$ or

$$
K_{2} \equiv \lim _{t \rightarrow 0} \frac{d^{2} \ln S(k, t)}{d t^{2}}=\frac{\left(D_{0} k^{2}\right)^{2}\left\{2 S_{D}(k)+c_{0} S(k) \int d \mathbf{r}[g(r)-1] e^{i \mathbf{k} \cdot \mathbf{r}} \hat{\mathbf{k}} \cdot \hat{\mathbf{r}} \beta \mathbf{F} \cdot \nabla(\beta \mathbf{F}) k^{-3}\right\}}{[S(k)]^{2}}
$$

As previously discussed by Ackerson ${ }^{7}$ and Phillies, ${ }^{26}$ the second cumulant of $S(k, t)$ gives extra information about the details of the intermacromolecular interactions.

\section{DISCUSSION}

The total effect of direct and hydrodynamic forces on the initial decay of $S(k, t)$ is given for $k \rightarrow 0$ by Eqs. (2.10) and (3.16):

$$
\begin{aligned}
K_{1}= & D_{0} k^{2}\left(1-\phi+4 \pi c_{0} \int_{0}^{\infty} r^{2} d r\left\{\frac{a}{r}[g(r)-1]-\frac{5}{2}\left(\frac{a}{r}\right)^{4} g(r)\right\}\right) \\
& -2 \lim _{t \rightarrow 0}\left\langle a_{-10} \sum_{i=1}^{N} e^{i \mathbf{r} \cdot x_{i}(0)} \int_{0}^{t} d s \mathbf{k} \cdot v_{B i}(s) \mathbf{k} \cdot \mathbf{v}_{I I}(t)\right\rangle \cdot(5.1)
\end{aligned}
$$

The final term is discussed elsewhere. ${ }^{25}$
The term $-\phi$ is a reference frame correction, reflecting the difference between diffusion relative to the solvent and diffusion relative to the fixed volume of the sample. From Eq. (3.15) and the Appendix, the reference frame correction depends on $H(k)$, whose presence has a simple physical interpretation. $S(\mathbf{k}, t)$ decays because scatterers move across the planes perpendicular to $\mathrm{k}$; as the scatterers move, they displace solvent, which flows backwards across the same planes, dragging other scatterers with it. If the scatterers are pointlike, the solvent backflow appears uniform to them. However, for a given fluctuation $a_{k}(0)$, the current of scatterers has on the average a spatial dependence like $\exp (i \mathrm{k} \cdot r)$, i.e., the direction of the solvent backflow reverses sign every half-wavelength along $k$. If a scatterer is of finite 
extent, different parts of it will experience different solvent backflow velocities. A rigid particle responds to the average backflow velocity, the average being giv en by $H(k)$. The presence of the reference frame correction thus depends on the size $r_{0}$ of the particle. For pointlike particles $\left(k r_{0} \ll 1\right)$, as have usually been examined in the past, $H(k)=1$. However, if one were to study the diffusion of a concentrated $(\phi>0.01)$ suspension of large $(Z 500 \AA)$ particles, the reference frame correction to $D_{m}$ might deviate substantially from its longwavelength $(k-0)$ form.

The decay of concentration fluctuations is known to be approximately exponential. From its initial slope, the decay constant of the exponential must be approximately

$$
\Gamma=\frac{D_{0} k^{2}(1-\phi)}{S(k)}+(\text { hydrodynamic corrections }) \text {. }
$$

Pusey ${ }^{19}$ argues that in the absence of hydrodynamic forces $\Gamma$ is determined solely by single-particle Brownian motion, i.e., that in the absence of hydrodynamic forces there exists a time scale between $t \gg \tau$ and the earliest time at which $\mathbf{v}_{I i}$ appreciably affects the decay of $a_{k}(t)$. Our conclusion here is that this time scale does not exist, in that $\mathrm{v}_{I i}$ has a major effect on $\dot{a}_{k}(t)$ as soom as $t \gg \tau_{B}$. The argument of Pusey is based on the definition of the diffusion coefficient

$$
2 \mathbf{D}=\int d t\left\langle\mathrm{v}_{i}(t) \mathrm{v}_{i}(t+\tau)\right\rangle
$$

and $\mathrm{Eq}$. (2.9) for the velocity. In combining Eqs. (5.3) and (2.9), Ref. 19 neglects cross terms between $v_{B 1}$ and $v_{I i}$. Since $\left(v_{B i}\right)^{2}$ on the average is much larger than
$\left(\mathbf{v}_{D i}\right)^{2}$, Ref. 19 concludes that $\left\langle\int \mathbf{v}_{B i}(t) \mathbf{v}_{B t}(t+\tau) d \tau\right\rangle$ is much larger than $\left\langle\int \nabla_{I i}(t) v_{I_{i}}(t+\tau) d \tau\right\rangle$, so that Eq. (5.3) is dominated by the $\left[\mathbf{v}_{B i}(t)\right]^{2}$ term. This conclusion does not appear to take into account the consideration that the correlation time $\tau_{B}$ of $\mathrm{v}_{B i}$ is much less than that of $\nabla_{I i}$. The integral in Eq. (5.3) is approximately a product of $\left[v_{i}(t)\right]^{2}$ and a correlation time $\tau_{B}$ or integration time $t$ so the fact that $\left(v_{B i}\right)^{2} \gg\left(v_{I i}\right)^{2}$ does not guarantee that $\left(v_{B i}\right)^{2} \tau_{B} \gg\left(v_{I i}\right)^{2} t$. Equations (3.9) and (3.13) show that the integrals over $v_{B}$ and $v_{I}$ actually contribute to the initial decay of $S(k, t)$ to a similar extent.

In a previous paper, one of $\mathrm{us}^{20}$ suggested that light scattering spectroscopy actually measures the singleparticle diffusion rather than pair diffusion, since in the correlation function

$G_{i}(\mathbf{r}, t)=\frac{1}{N} \sum_{j=1}^{N}\left\langle\delta\left(\mathbf{r}+\left[\mathbf{r}_{j}(0)-\mathbf{r}_{i}(0)\right]-\left[\mathbf{r}_{i}(t)-\mathbf{r}_{i}(0)\right]\right)\right\rangle$

the time dependent term is $r_{i}(t)-r_{i}(0)$. The position of each particle at time $t$ is associated only with its own position at the previous time 0 . However, the right hand side of Eq. (5.4) also depends on $\mathbf{r}_{f}(0)$, so $G_{i}(r, t)$ includes any correlations between the displacement of particle $i$ during $(0, t)$ and the positions of other particles at $t=0$. In Sec. III, this correlation is shown to be significant; the presence of these correlations means that $\mathrm{G}_{i}(r, t)$ is sensitive to mutual diffusion rather than to solute self-diffusion.

The second cumulant of $S(\mathbf{k}, t)$ was previously obtained by Ackerson $^{6}$ as

$$
K_{2}=\frac{D_{0}^{2} k^{4}\left\{S(k)-1+S(k) \beta c_{0} \int d \mathbf{r} g(r)[1-\cos (\mathbf{k} \cdot \mathbf{r})](\mathbf{k} \cdot \nabla)^{2} u(r) / k^{4}\right\}}{[S(k)\}^{2}}
$$

Noting that $u(r) \equiv W_{i j}(r)$ is only a function of $|\mathrm{r}|$, $(\mathbf{k} \cdot \nabla) u=k \cdot r(8 u / \partial r)$. The integral in $\mathbf{E q} .(5.5)$ is very similar, not to Eq. (4.9), but to Eq. (4.4), suggesting that the computation in Ref. 6, like that leading to Eq. (4.4), neglects terms in $\partial v_{I i} / \partial t$.

\section{APPENDIX: HYDRODYNAMIC INTERACTION TENSOR FOR PARTICLES IN AN ENCLOSED FINITE VOLUME}

We here justify our assertion that on the average

$$
\int_{\mathbf{v}} d \mathbf{r}_{\mathbf{i}}\left[\hat{\mathbf{k}} \cdot \mathbf{b}_{i j}^{\prime} \cdot \hat{\mathbf{k}} e^{i \mathbf{r} \cdot \mathbf{r}_{i j}}+\phi_{j} H(k)\right]=0,
$$

where $V$ is the container volume, $\hat{\mathrm{k}}$ denotes the unit vector of $\mathbf{k}, \phi_{j}$ is the volume fraction relative to the container of particle $j$ (which is taken to drive the flow), and $H(k)$ is the spatial Fourier transform of the hydrodynamic excluded volume of particle $j$.

The physical basis of Eq. (A1) is the assumption that, for a finite volume of an incompressible fluid, the total volume flow (of solute plus solvent) across any plane must vanish. The assumption for closed containers may be stated mathematically as

$$
\int_{s_{i}} d \mathbf{r}_{i} \hat{\mathbf{s}} \cdot \mathbf{b}_{i j}^{\prime} \cdot \mathbf{v}_{j}=-C_{j}\left(r_{i}\right) \hat{\mathbf{s}} \cdot \mathbf{v}_{j}, \quad\left(j \in S_{i}\right),
$$

where $S$ is a plane across the container with normal $\hat{\mathbf{s}}$, $C_{j}\left(r_{i}\right)$ is the cross section of particle $j$ in $S$, and the sum on the rhs of Eq. (A3) is restricted to particles lying partially within $S$. A moving particle $j$ of volume $V_{j}$ and location $r_{j}$ excites at the point $r_{i}$ in the fluid a flow $v_{i}$ :

$$
\mathbf{v}_{i}=\mathbf{b}_{i j}^{\prime} \cdot \mathbf{v}_{j},
$$

where $\nabla_{j}$ is the velocity of particle $j$ and $b_{i j}^{\prime}$ is a function of the positions of all the particles.

For $\hat{\mathbf{k}} \| \hat{\mathbf{s}}, \exp (i \mathbf{k} \cdot r)$ is a constant within $S_{i}$, so

$$
\int_{s_{i}} d \mathbf{r}_{i} \hat{\mathbf{k}} \cdot \mathbf{b}_{i j}^{\prime} \cdot \mathbf{v}_{j} e^{i \mathbf{k}\left(\mathbf{r}_{i}-\mathbf{r}_{j}\right)}=-e^{i \mathbf{k} \cdot\left(\mathbf{r}_{i}-\mathbf{r}_{j}\right)} C_{j}\left(\mathbf{r}_{i}\right) \hat{\mathbf{k}} \cdot \mathbf{v}_{j}
$$

Since particle $j$ may be of finite extent, $j \in S_{i}$ does not imply that $\mathbf{r}_{j}$ itself lies in $S_{i}$. The final step is to integrate over all planes $S_{i}$ :

$$
\int_{V} d r_{i} \hat{\mathbf{k}} \cdot \mathbf{b}_{i j}^{\prime} \cdot \hat{\mathbf{k}} e^{i \mathbf{k} \cdot \mathbf{r}_{i j}\left(\hat{\mathbf{k}} \cdot \mathbf{v}_{j}\right)=-V_{j} H(k)\left(\hat{\mathbf{k}} \cdot \mathbf{v}_{j}\right),}
$$

where $r_{i j}=r_{i}-r_{j}$. On the lhs (neglecting points near 
the walls), the only tensors available to form $b_{i j}$ are I and $r r$, so by symmetry only the $k$ component of $\nabla_{j}$ contributes to the integral. On the rhs, since $C_{1}$ is implicitly only a function of $\mathbf{r}_{i j}$, we define

$$
\int_{v} d \mathbf{r}_{i} e^{i \mathbf{r} \cdot \mathbf{r}_{i}} ; C_{j}\left(\mathbf{r}_{i j}\right)=V_{j} H(k),
$$

where $H(0) \equiv 1$. Defining $\phi_{j}=V_{j} / V$, since the rhs of Eq. (A5) is independent of $r_{i}$ and Eq. (A5) is true for arbitrary $\left(\hat{\mathbf{k}} \cdot \mathbf{v}_{j}\right)$ :

$$
\int_{v} d \mathbf{r}_{i}\left[\hat{\mathbf{k}} \cdot \mathbf{b}_{i j}^{\prime} \cdot \hat{\mathbf{k}} e^{i \mathbf{k} \cdot \mathbf{x}_{i j}}+\phi_{j} H(k)\right]=0,
$$

where $r_{i}$ is now an arbitrary constant vector.

\footnotetext{
${ }^{1}$ A. R. Altenberger and J. M. Deutch, J. Chem. Phys. 59, 894 (1973).

${ }^{2}$ G. D. J. Phillies, J. Chem. Phys. 60, 976 (1974).

${ }^{3}$ G. D. J. Phillies, J. Chem. Phys. 60, 984 (1974).

${ }^{4}$ (a) S. S. Alpert and G. Banks, Biophys. Chem. 4, 287 (1976);

(b) G. D. J. Phillies, G. B. Benedek; and N. A. Mazer, J. Chem. Phys. 65, 1883 (1976).

${ }^{5}$ A. R. Altenberger, Chem. Phys, 15, $242(1976)$

${ }^{6}$ B. J. Ackerson, J. Chem. Phys. 64, 242 (1976).

'B. J. Ackerson, J. Chem. Phys, 69, 684 (1978).
}

${ }^{8}$ S. A. Allison, E. L. Chang, and J. M. Schurr, Chem. Phys. 38,29 (1979).

${ }^{9}$ A. R. Altenberger, J. Chem. Phys. 70, 1994 (1979).

${ }^{10} \mathrm{~J}$. L. Anderson and C. C. Reed, J. Chem. Phys. 64, 3240 (1976).

${ }^{11} \mathrm{G}$. K. Batchelor, J. Fluid Mech. 74, 1 (1976).

${ }^{12}$ (a) B. U. Felderhof, Physica (Utrecht) A 89, 373 (1977); (b) B. U. Felderhof, J. Phys. A 11, 929 (1978).

${ }^{13} \mathrm{~S}$. Harris, J. Phys. A 10, 1905 (1977).

${ }^{14}$ W. Hess and R. Klein, Physica (Utrecht) A 85, 509 (1976).

${ }^{15} \mathrm{G}$. Jacobs and S. Harris, Chem. Phys. Lett. 53, 115 (1978).

${ }^{16}$ W. K. Lee and J. M. Schurr, Chem. Phys, Lett. 38, 71 (1976).

${ }^{17}$ G. D. J. Phillies, J. Chem. Phys. 62, 3925 (1975).

${ }^{18}$ G. D. J. Phillies, J. Chem. Phys. 65, 4334 (1976).

${ }^{19}$ P. N. Pusey, J. Phys. A 8, 1433 (1975).

${ }^{20} \mathrm{P}$. R. Wills, J. Chem. Phys. 70, 5865 (1979).

${ }^{21}$ G. D. J, Phillies, J. Chem. Phys. 67, 4690 (1977).

${ }^{22}$ See W. Hess and R. Klein, Physica (Utrecht) A 85, 509 (1976) for an exception.

${ }^{23}$ J. G. Kirkwood, R. L. Baldwin, P, J. Dunlop, L. J. Gosting, and G. Kegeles, J. Chem. Phys. 33, 1505 (1960).

${ }^{24}$ R. S. Adler, J. Chem. Phys. 69, 2849 (1978).

${ }^{25}$ G. D. J. Phillies, J. Chem. Phys. 74, 2436 (1981). For an alternate calculation, giving an inequivalent result, see B. J. Ackerson, J. Chem. Phys. 68, 684 (1978); W. Dieterich and I. Peschel, Physica (Utrecht) A 95, 208 (1979); J. A. Marqusee and J. M. Deutch, J. Chem. Phys. 73, 5397 (1980); or J. A. Marqusee, Ph.D. Thesis, M.I.T., 1981.

${ }^{26}$ G. D. J. Phillies, Macromolecules 9, 447 (1976). 